\title{
Bilateral optic neuritis as a first presentation of lymph node tuberculosis
}

\author{
Authors \\ Ashvini Keshavan, MRCP PhD $1,2+$ \\ a.keshavan@ucl.ac.uk \\ Lisa McAnena, MCh MRCPI FRCSI (Ophth)³ Imcanena@yahoo.co.uk \\ James F Acheson, MRCP FRCOphth $2,3 \quad$ james.acheson@nhs.net \\ Helen Booth, FRCP 4 helen.booth4@nhs.net \\ Gordon T Plant, MD FRCP FRCOphth¹‥plant@ucl.ac.uk \\ Zhaleh Khaleeli, MRCP PhD² z.khaleeli@nhs.net
}

${ }^{+}$Corresponding author: Dr Ashvini Keshavan, Dementia Research Centre, Box 16, National Hospital for Neurology and Neurosurgery, Queen Square, London WC1N 3BG, UK

E-mail: a.keshavan@ucl.ac.uk

\section{Affiliations}

1. UCL Queen Square Institute of Neurology, University College London, London, UK

2. National Hospital for Neurology and Neurosurgery, Queen Square, London, UK

3. Moorfields Eye Hospital, City Road, London, UK

4. North Central London Tuberculosis Service, Whittington Health NHS Trust and Department of Respiratory Medicine, University College London Hospitals, London, UK

\section{ORCiD}

Ashvini Keshavan: 0000-0003-1043-5721

Lisa McAnena: 0000-0002-8025-6203 


\begin{abstract}
Tuberculosis (TB) may affect the nervous system in many ways. Here we describe a case of lymph node TB that first presented as a bilateral optic neuritis in an immunocompetent adolescent. We discuss the importance of a detailed history to identify features inconsistent with a diagnosis of optic neuritis as seen in multiple sclerosis and other immune-mediated disorders. In this case several unusual features prompted further investigation, including transient visual obscurations without raised intracranial pressure, prominent disc swelling and the absence of laboratory findings to support an immune-mediated optic neuritis. Whole body PET/MR imaging identified widespread mediastinal and supraclavicular lymphadenopathy. Despite the patient having no known TB contact, a negative interferon gamma release assay and a normal chest X-ray, mycobacterial culture of a targeted lymph node biopsy confirmed tuberculosis.
\end{abstract}

\title{
Clinical summary
}

A 16-year-old female student presented with a three-week history of progressive left eye vision loss, starting in the central visual field and expanding slowly over the first two weeks. In the first two days she had a mild left supraorbital headache; for the first week she had pain on eye movement and felt her upper left lid was swollen. She mentioned transient loss of left eye vision provoked by standing up from bending forward.

On further questioning she had at least three prior episodes of left eye vision loss within the preceding 9 months, with possible incomplete recovery between, but had not presented to medical services at those times. The first was a painless transient 
darkening of the whole left eye vision without any obvious precipitant, resolving spontaneously after a few minutes. The second and third episodes occurred a month and four months later respectively, each with a week of pain on eye movement, followed by patchy loss of vision in the left eye, that began to improve after a further week but did not normalise. There was no history of photopsia or red eye. She had no systemic or other neurological symptoms, no other past medical history, and took no medications. There was no relevant family history and no contacts with cough or fever. She did not smoke or take alcohol or recreational drugs.

She was born in Italy and received all the usual childhood immunisations given there, which did not include Bacille Calmette-Guérin (BCG) vaccination for Mycobacterium tuberculosis (TB). She had travelled thrice to Bangladesh, firstly at age 5 , again at age 7 when she stayed there for 18 months, and most recently at age 14 when she stayed there for 2 weeks. She migrated to the UK from Italy as a teenager with her family. There was no known TB contact.

On examination, distance visual acuity corrected with glasses was $6 / 5$ on the right and $6 / 9$ on the left and did not improve with pinhole. Ishihara colour vision test scores were $17 / 17$ on the right and $15 / 17$ on the left. Humphrey visual fields showed an enlarged blind spot and restricted temporal field on the left, but also subtle superior and inferior field deficits on the right. Slit lamp examination revealed normal anterior segments without evidence of intraocular inflammation; the right disc appeared normal but the left was swollen. 
Optical coherence tomography (OCT) confirmed swelling of the left optic nerve head and thinning of the retinal ganglion cell layer temporal to the left macula (Figure 1A). MRI brain and orbit showed swelling and enhancement of both the anterior left and the pre-chiasmal right optic nerves, without other intracranial or meningeal lesions or enhancement (Figure 2). Lumbar puncture opening pressure was $19.5 \mathrm{~cm}$ of water; CSF was acellular, with normal protein, glucose and cytology, and negative for viral PCR, oligoclonal bands and acid-fast bacilli staining. Blood tests showed a raised erythrocyte sedimentation rate of $27 \mathrm{~mm} / \mathrm{hr}$ and positive Varicella zoster lgG, indicating past infection or immunisation. The following blood tests were normal or negative: full blood count, urea and electrolytes, liver function tests, bone profile, Creactive protein, vitamin B12, ANA, ENA, dsDNA, ANCA, antibodies to aquaporin-4 and myelin oligodendrocyte glycoprotein, HIV 1 and 2 antibodies and p24 antigen, serology for syphilis and toxoplasma, and QuantiFERON-TB® Gold in-tube (QFT-GIT) interferon gamma release assay (IGRA). A chest radiograph was normal.

We initially diagnosed bilateral optic neuritis and treated with oral methylprednisolone $500 \mathrm{mg} /$ day for 5 days, after which she had a good recovery of visual function. As there were atypical features for immune-mediated optic neuritis of any cause, a whole-body FDG-PET/MRI scan was requested; this showed multiple FDG-avid enlarged mediastinal and supraclavicular lymph nodes, with no other lung or visceral lesions. While awaiting lymph node biopsy she re-presented, two months after the previous steroid pulse (i.e. 12 months after symptom onset), with right eye pain on movement and subjective vision loss. Although there was no objective deficit of visual acuity or colour vision, OCT showed swelling of the superior nasal aspect of 
the right optic nerve head, with atrophy of the left optic nerve head; left sided ganglion cell layer thinning had also progressed (Figure 1B).

A supraclavicular lymph node core biopsy showed necrotising granulomata, smearnegative but subsequently culture-positive for a fully sensitive Mycobacterium tuberculosis. She was treated with quadruple therapy (rifampicin, isoniazid, pyrazinamide and ethambutol) without further steroids. Medication-induced hepatitis necessitated a pause in treatment after one week, but after her liver function tests normalised, she started an alternative TB induction drug regime (rifampicin, isoniazid and levofloxacin). At six weeks after re-starting treatment (14 months after symptom onset), she reported no further relapses. Visual acuity was $6 / 5$ on the right and $6 / 6$ on the left with colour vision 13/13 bilaterally, stable visual fields and OCT findings.

\section{Discussion}

Unilateral transient visual obscurations (TVO) imply unilateral intermittent disc hypoperfusion. This is seen most commonly in raised intracranial pressure and is likely to be due to a mismatch between the high metabolic demand of the nerve, and lower perfusion arising from local venous hypertension. Typically, the symptom occurs if a further transient increase in venous pressure, e.g. on bending forward, is followed by a transient fall in arterial perfusion, e.g. on standing up. Intra-ocular pressure also influences optic nerve and retinal perfusion, but changes little with posture. In our patient's case the TVO may indicate that the disc swelling was at least partly due to venous congestion; similar presentations can occur in intrinsic lesions such as optic nerve glioma, and extrinsic lesions such as nerve sheath meningioma. 
We performed a whole-body PET/MRI scan because our patient had several unusual features for an immune-mediated optic neuritis as occurs in multiple sclerosis (MS) or neuromyelitis optica (NMO); these included prominent disc swelling, TVO without raised intracranial pressure and normal/negative results on relevant blood and CSF tests. Although PET/MRI is readily available in our institution, it would also have been reasonable, in case of reduced access to PET, to perform a contrast-enhanced CT of the chest, abdomen and pelvis, to screen for the presence of a systemic inflammatory condition like sarcoidosis. Given the clinical features, response to corticosteroids and the PET/MR findings, lymphomatous involvement of the optic nerve was a possible diagnosis; appropriate anti-tuberculous treatment (ATT) was started only once a positive microbiological diagnosis was obtained.

CNS involvement overall occurs in $1-5 \%$ of people with TB [1], and ocular involvement in 1-18\% [2]. Globally, there are no published incidence or prevalence figures for TB optic neuropathy as a proportion of people with TB. It is reported most commonly in residents or travellers to Asia, and is bilateral in $25 \%$ of cases. The commonest presenting features are of anterior optic neuritis, perineuritis and neuroretinitis. In optic neuritis there is painful vision loss particularly on eye movement, and disc swelling may be observed if the optic nerve head is involved. In optic perineuritis the pain may be particularly severe, the vision loss tends to be more in the peripheral than central field, and neuroimaging may demonstrate contrast enhancement in the optic nerve sheath. In neuroretinitis there is disc swelling and the appearance of a macular star resulting from subretinal exudate (see Practical Neurology Volume 20, Issue 6 [3]). Other types of optic nerve involvement 
in TB include optic nerve head tuberculomas, which may be sometimes be observed directly. Retrobulbar optic neuritis, compressive and anterior ischaemic optic neuropathic presentations are less common.

TB optic neuropathy usually also presents with uveitis (in $89 \%$ of cases), other forms of ocular inflammation such as vasculopathy, or signs of TB meningitis, but the absence of these features does not exclude the diagnosis. Evidence of extra-ocular TB, including meningitis, was reported in about $37 \%$ of cases in a series of 49 patients from North America, Asia, Africa and Europe [4]. Optic neuritis is unusual as a presenting feature of TB in immunocompetent people, where case reports describe presumptive diagnoses, in a close contact of a confirmed TB case [5] or in the context of concomitant culture-positive chronic TB meningitis [6]. Biopsy of the optic nerve itself is not undertaken to confirm the diagnosis, but optic nerve sheath biopsy has demonstrated $M$. tuberculosis in at least one reported case of a French Caucasian patient who had no obvious TB risk factors [7]. These various reports suggest immunocompetent individuals make a good visual recovery with ATT alone (without corticosteroids), but significant symptomatic improvement may occur with corticosteroid treatment alone, before the diagnosis is established, as indeed was the case with our patient. UK NICE guidance recommends corticosteroids as adjunctive treatment for CNS and pericardial TB [8] and they may be used at discretion in other situations including paradoxical reactions to ATT.

A positive IGRA and/or tuberculin skin test (TST) are highly specific but only moderately sensitive to the presence of a cellular immune response to $M$. tuberculosis. They are indirect markers of previous exposure but cannot accurately 
differentiate between latent or active infection. UK ophthalmology services have widely adopted IGRA to investigate possible ocular TB, as they are easier to perform, more specific (no cross reactivity with non-tuberculous mycobacteria or BCG) and more sensitive for active TB (80\% and $81 \%$ for QFT-GIT and T-SPOT respectively) compared with TST (65\%) [9]. However, our patient was IGRA negative (despite absence of factors like older age and lower peripheral blood lymphocyte count that are known to lead to false negative IGRA [10]), highlighting that a negative IGRA cannot exclude TB.

Drug treatment for tuberculous optic neuritis involves combination therapy similar to that used for CNS TB in other locations, taking into account geographical resistance patterns. Importantly, ethambutol itself can cause optic neuropathy (which is usually painless and bilateral), with prevalence estimated at 0.7 to $1.3 \%$ in patients treated with it for TB [11]. Prevalence increases with drug dosage but idiosyncratic reactions may also occur regardless of dose. Both the American Thoracic Society/Centers for Disease Control and Prevention/Infectious Diseases Society of America [12] and Royal College of Ophthalmologists guidelines [13] stipulate a baseline ocular assessment should always be undertaken prior to starting ethambutol, but the former also advocate monthly subjective vision symptom assessment and colour vision testing, while the latter suggest monitoring of self-reported symptoms, and to stop the drug immediately if new symptoms are experienced. In those at high risk for ethambutol toxicity (e.g. renal impairment), particularly in settings where close ophthalmic follow-up cannot be offered, we suggest that ethambutol should be avoided.

\section{Key points}


- TB can damage the optic nerves. It typically presents as an anterior optic neuritis (subacute pain, visual loss and optic disc swelling).

- In contrast to immune mediated anterior optic neuritis (e.g. in MS or NMO), TB optic neuritis may cause transient visual obscurations.

- Even without evidence of intra-ocular inflammation, TB should be considered as a potential cause of optic neuritis which is atypical for immune-mediated disorders, particularly in individuals with risk factors for TB exposure.

- TB interferon gamma release assays and tuberculin skin tests can be negative in cases of active TB disease.

\section{Acknowledgements}

We are grateful to the patient and her parents for permission to publish, Miss Victoria Nowak for helping with OCT images, Dr Veronica White for providing care in the TB service, and Dr Stephen Morris-Jones for microbiology advice.

\section{Funding}

No specific funding was received for this study.

\section{Contribution statement}

AK, GTP and ZK conceptualised the study. LM and JA acquired and analysed clinical data. AK performed the literature review, acquired and analysed clinical data, and drafted the manuscript. All other authors revised the manuscript critically for important intellectual content. All authors gave final approval of the submitted manuscript. 


\section{Conflicts of interest}

Unrelated to this work, AK receives research funding from the Selfridges Foundation (Weston Brain Institute Grant UB170045).

All other authors declare no conflicts of interest. 


\section{Figure 1}

Optical coherence tomography on presentation at 9 months $(A)$ showing very subtle disc swelling on the right (shown by subtle increase in retinal nerve fibre layer green arrow) although disc appearance in fundal photographs was normal. On the left there was marked disc swelling (see fundal photograph, and the patient's retinal nerve fibre layer volume at the disc was far above the normal range - white arrow), but there was already evidence of atrophy of the ganglion cell layer around the macula (shown by dark red areas on the colour map), demonstrating this was a longstanding process. This demonstrates the mismatch between the optic disc swelling (due to a combination of axonal distension and tissue oedema, visible in the sub-retinal layer) and thinning of the ganglion cell layer due to extensive axonal loss from the same population of neurons. At 12 months after symptom onset (B), the right optic disc was more swollen (increase in retinal nerve fibre layer volume green arrow) and the swelling on the left had resolved with the development of disc atrophy (loss of retinal nerve fibre layer - black arrow), and further loss of ganglion cell layer volume at the macula (increase in dark red areas on colour map). 

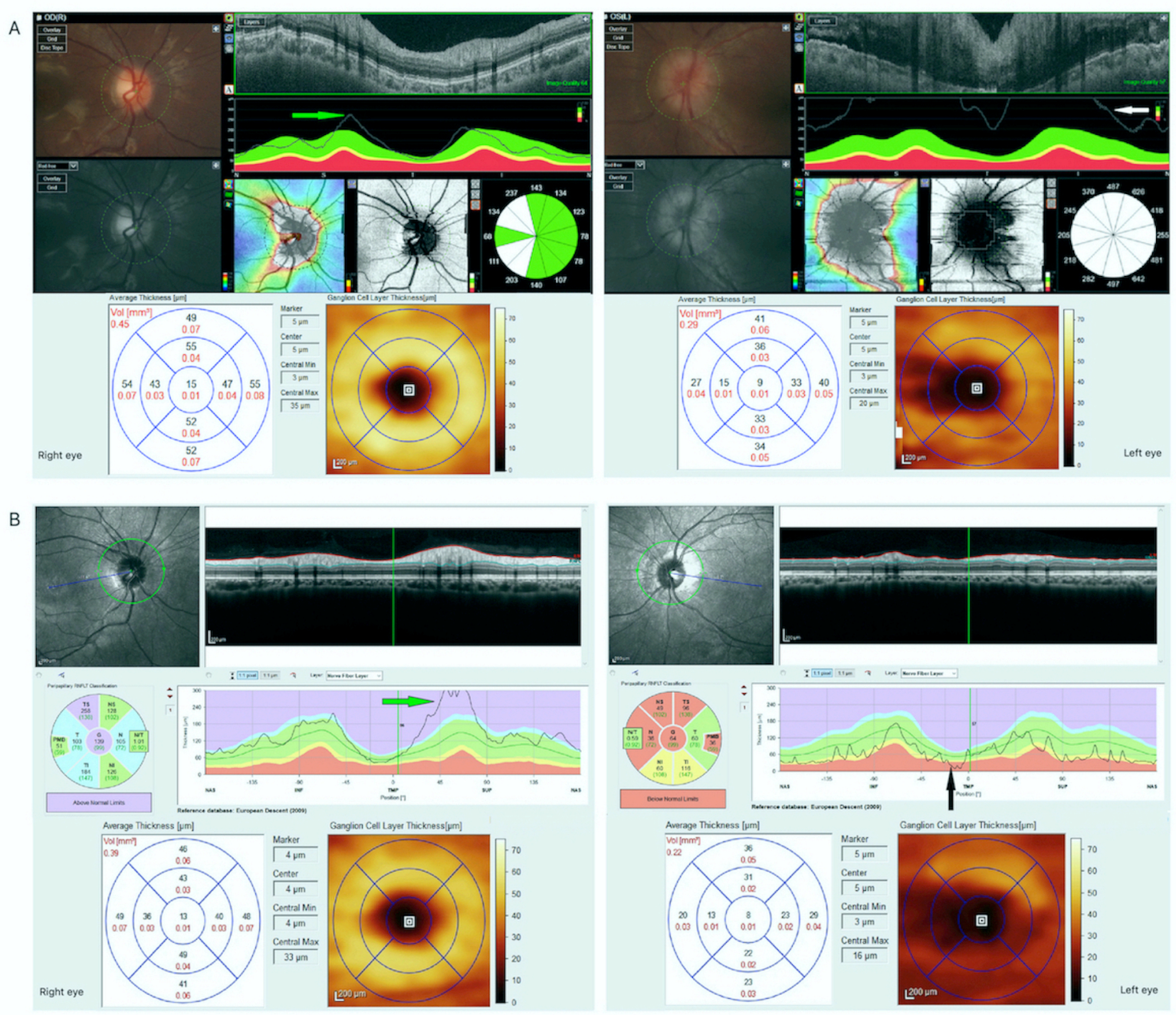


\section{Figure 2}

MRI brain and orbits T1 fat-suppressed post-contrast on presentation at 9 months, showing enhancement of the anterior left optic nerve on the axial view $(A)$ with posterior flattening of the left globe (cyan arrow) and effacement of left nerve sheath CSF low T1 signal on the coronal view from left optic nerve swelling swelling (magenta arrow). There was also enhancement of the pre-chiasmatic right optic nerve, as demonstrated on an axial view (C: white arrow).

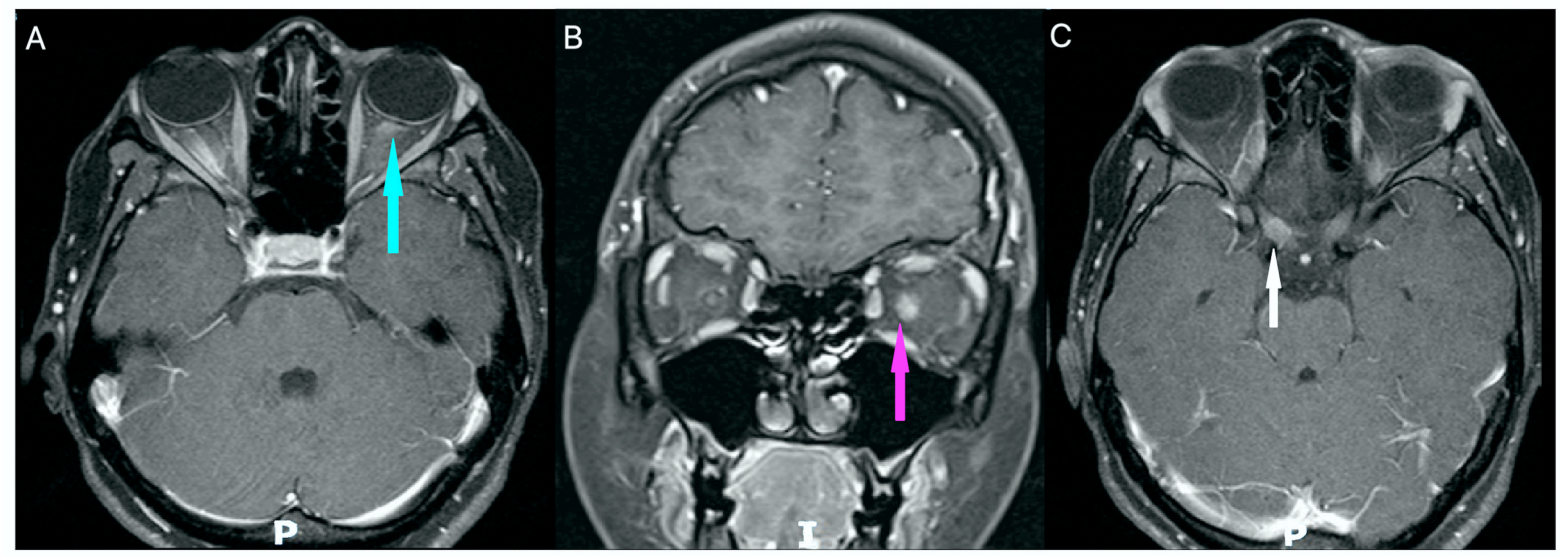




\section{Recommended reading}

NICE. Tuberculosis. NICE Guideline 33. London, 2019 [updated 12/09/2019.

Available from: http://www.nice.org.uk/guidance/ng33.

Davis EJ, Rathinam SR, Okada AA, Tow SL, Petrushkin H, Graham EM, et al. Clinical spectrum of tuberculous optic neuropathy. J Ophthalmic Inflamm Infect. 2012;2(4):183-9. 


\section{References}

1. Garg RK. Central nervous system tuberculosis: An overview 2020 [updated 10/12/2020. Available from: https://www.uptodate.com/contents/central-nervoussystem-tuberculosis-an-overview.

2. Rathinam SR. Tuberculosis and the eye 2019 [updated 28/10/2019. Available from: https://www.uptodate.com/contents/tuberculosis-and-the-eye.

3. Lueck CJ. Neuroretinitis: a tricky mimic. Pract Neurol. 2020;20(6):430-2.

4. Davis EJ, Rathinam SR, Okada AA, Tow SL, Petrushkin H, Graham EM, et al. Clinical spectrum of tuberculous optic neuropathy. J Ophthalmic Inflamm Infect. 2012;2(4):183-9.

5. Norazizah MA, Wan Hazabbah WH, Rohaizan Y, Shatriah I. Isolated optic neuritis secondary to presumed tuberculosis in an immunocompetent child. Med $\mathrm{J}$ Malaysia. 2012;67(1):102-4.

6. Narayanan S, Prakash D, Subramaniam G. Bilateral primary optic neuropathy as the presenting manifestation of tuberculosis in an immunocompetent patient. IDCases. 2019;18:e00579.

7. Aupy J, Vital A, Rougier MB, Gradel A, Meissner W, Marchal C, et al. Presumed tuberculous retrobulbar optic neuritis: a diagnosis challenge. J Neurol. 2015;262(2):481-4.

8. NICE. Tuberculosis. NICE Guideline 33. London2019 [updated 12/09/2019. Available from: http://www.nice.org.uk/guidance/ng33.

9. Sester M, Sotgiu G, Lange C, Giehl C, Girardi E, Migliori GB, et al. Interferongamma release assays for the diagnosis of active tuberculosis: a systematic review and meta-analysis. Eur Respir J. 2011;37(1):100-11.

10. Yamasue M, Komiya K, Usagawa Y, Umeki K, Nureki SI, Ando M, et al. Factors associated with false negative interferon-gamma release assay results in patients with tuberculosis: A systematic review with meta-analysis. Scientific reports. 2020;10(1):1607.

11. Chamberlain PD, Sadaka A, Berry S, Lee AG. Ethambutol optic neuropathy. Curr Opin Ophthalmol. 2017;28(6):545-51.

12. Nahid P, Dorman SE, Alipanah N, Barry PM, Brozek JL, Cattamanchi A, et al. Official American Thoracic Society/Centers for Disease Control and Prevention/Infectious Diseases Society of America Clinical Practice Guidelines:

Treatment of Drug-Susceptible Tuberculosis. Clin Infect Dis. 2016;63(7):e147-e95. 13. Group QaS. RCOphth Statement on Ethambutol Toxicity: Royal College of Ophthalmologists; 2017 [Available from: https://www.rcophth.ac.uk/2017/10/rcophthstatement-on-ethambutol-toxicity/. 